\title{
Block Matrix Representation of a Graph Manifold Linking Matrix Using Continued Fractions
}

\author{
Fernando I. Becerra López, Vladimir N. Efremov, Alfonso M. Hernandez Magdaleno \\ Mathematics Department, CUCEI, University of Guadalajara, Guadalajara, Mexico \\ Email: mail@ferdx.com, efremov@cencar.udg.mx, 137mag@gmail.com
}

Received 19 April 2014; revised 27 May 2014; accepted 6 June 2014

Copyright (C) 2014 by authors and Scientific Research Publishing Inc. This work is licensed under the Creative Commons Attribution International License (CC BY). http://creativecommons.org/licenses/by/4.0/

(c) $\underset{\mathrm{EY}}{0}$ Open Access

\begin{abstract}
We consider the block matrices and 3-dimensional graph manifolds associated with a special type of tree graphs. We demonstrate that the linking matrices of these graph manifolds coincide with the reduced matrices obtained from the Laplacian block matrices by means of Gauss partial diagonalization procedure described explicitly by $W$. Neumann. The linking matrix is an important topological invariant of a graph manifold which is possible to interpret as a matrix of coupling constants of gauge interaction in Kaluza-Klein approach, where 3-dimensional graph manifold plays the role of internal space in topological 7-dimensional BF theory. The Gauss-Neumann method gives us a simple algorithm to calculate the linking matrices of graph manifolds and thus the coupling constants matrices.
\end{abstract}

\section{Keywords}

\section{Graph Manifolds, Continued Fractions, Laplacian Matrices, Kaluza-Klein}

\section{Introduction}

Graphs can serve as a universal remedy for the codification and classification of topological spaces, matrices, dynamical systems, etc. In this article, we consider the following question: how the topological invariants of manifold corresponding to a tree graph (graph manifold) can be calculated using the method of Gauss-Neumann partial diagonalization of Laplacian matrix defined for the same graph. This calculation can be useful in multidimensional models of Kaluza-Klein type, where coupling constants of gauge interactions are simulated by the rational linking matrices of the internal space [1]. We constructed various models [2] [3] where the role of internal spaces is played by a specific family of 3-dimensional graph manifolds, whose rational linking matrices 
describe the hierarchy of gauge coupling constants of the real universe. The basic structure blocks of these graph manifolds are Seifert fibered Brieskorn homology spheres, defined as the link of Brieskorn singularity

$\Sigma(\underline{a}):=\Sigma\left(a_{1}, a_{2}, a_{3}\right):=\left\{z_{1}^{a_{1}}+z_{2}^{a_{2}}+z_{3}^{a_{3}}=0\right\} \cap S^{5}$, with $a_{1}, a_{2}, a_{3}$ pairwise relatively prime positive numbers [4]. Bh-spheres belong to the class of Seifert fibered homology spheres (Sfh-spheres). On each of these manifolds, there exists a Seifert fibration with unnormalized Seifert invariants $\left(a_{i}, b_{i}\right)$ subject to

$e(\Sigma(\underline{a}))=\sum_{i=1}^{3} b_{i} / a_{i}=-1 / a$, where $a=a_{1} a_{2} a_{3}$ and $e(\Sigma(\underline{a}))$ is its rational Euler number, the well known topological invariant of a Bh-sphere. The topological operation known as plumbing [5] is used to past together Bh-spheres according to plumbing diagrams (Figure 4) which can be transformed in plumbing graphs (Figure 3). This type of graphs and their Laplacian block matrices is the main object of attention in this article.

The paper is organized as follows. In section 2 we define the type of tree graphs considered in this paper (plumbing graphs) and Laplacian matrices for these plumbing graphs. We recall also the Gauss-Neumann method of partial diagonalization by means of which we obtain the reduced rational tridiagonal matrix for each plumbing graph. In section 3 we construct graph manifolds codified by the plumbing graphs defined in section 2 and calculate the main topological invariant for these 3-dimensional manifolds, namely rational linking matrix. Then we demonstrate that the linking matrices of these graph manifolds coincide with the reduced matrices obtained from the Laplacian block matrices by means of Gauss-Neumann partial diagonalization procedure. Finally, we conclude formulating our main results and considering an example of their application for the topological field theory.

\section{Block Matrix Representation for a Graph $\Gamma_{p}$ of Tree Type}

We begin from the definition of graph $\Gamma$ as a finite one-dimensional simplicial complex, which does not contain multiple edges and loops, i.e. we consider only the graph of tree type. An integer weight $e_{i}$ is assigned to each vertex of $\Gamma$. Vertices with at least three edges are called nodes. For simplicity we shall use graphs with nodes of minimal valence $(n=3)$ only (a generalization to $n \geq 3$ is obvious). Suppose that the set of nodes $\mathcal{N}$ of the graph $\Gamma$ is non-empty. Considering the graph $\Gamma$ as a one-dimensional simplicial complex, we take the complement $\Gamma-\mathcal{N}$. This complement is the disjoint union of straight line segments which are the maximal chains of $\Gamma$. Figure 1 shows a maximal internal chain $\left\{v_{1}, \cdots, v_{k}\right\}$ of length $k$ between two nodes $N^{I}$ and $N^{J}$, with weights $\left\{e_{1}, \cdots, e_{k}\right\}$ embedded in a tree graph $\Gamma$. The chain is maximal because it cannot be included in some larger chain. Figure 2 shows a maximal terminal chain $\left\{v_{1}, \cdots, v_{k}\right\}$ of length $k$ also with weights $\left\{e_{1}, \cdots, e_{k}\right\}$.

In this paper we shall considered only the simplest type of graphs which are called plumbing graphs. An example of plumbing graph $\Gamma_{p}$ is given in Figure 3. This type of graphs is used to codify the plumbing graph manifolds [5] which will be constructed in the following section, where it will be clear why weights of plumbing graph are called Euler numbers. In Figure 3 the Euler numbers $\epsilon_{i}^{I}$ and $e_{i}^{I}$ decorate the vertices with valence 1 and 2 . The nodes are marked by $N^{I}$ with $I=1, \cdots, R$ and form a straight line or chain structure. We associate
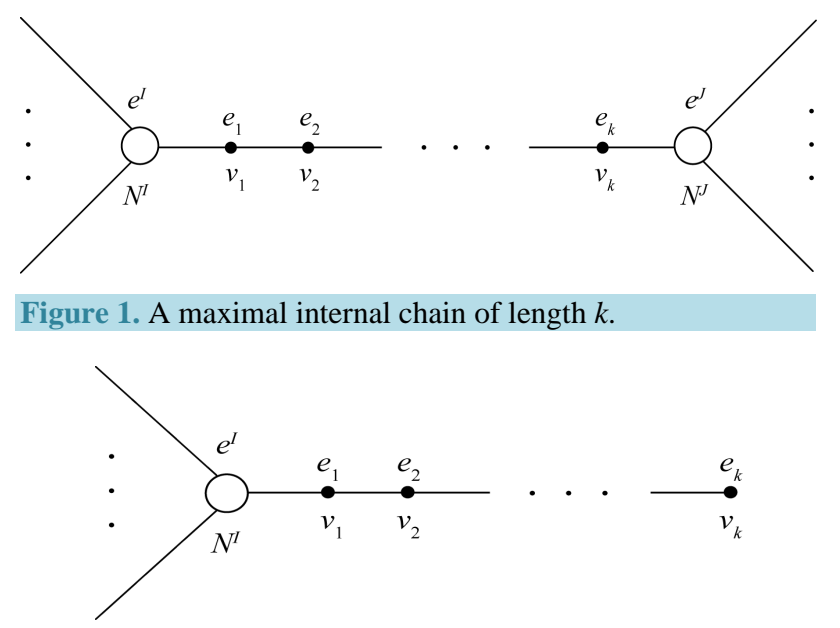

Figure 2. A maximal terminal chain of length $k$. 


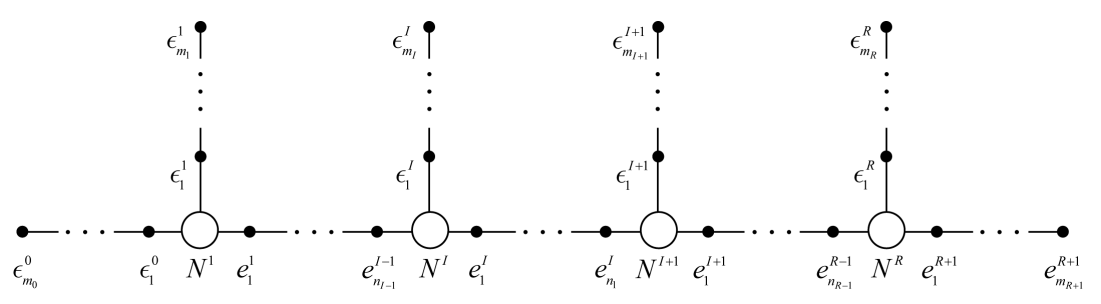

Figure 3. The plumbing graph $\Gamma_{p}$.

to each node a weight equal to zero that is connected with using of the unnormalized Seifert invariants for Bh-spheres, which are the block elements for the construction of graph manifolds [6].

Now let's define a Laplacian matrix for the plumbing graph $\Gamma_{p}$ as follows:

$$
Q^{A B}\left(\Gamma_{p}\right)= \begin{cases}e_{A}, & \text { if } A=B ; \\ -1, & \text { if } A \neq B \text { and } v_{A} \text { is connected to } v_{B} \text { by an edge; } \\ 0, & \text { otherwise, }\end{cases}
$$

with integer numbers $e_{A}$ corresponding to each vertex $v_{A}$. This is a tridiagonal block matrix containing all the information about the graph $\Gamma_{p}$. The $I$-th fragment of the matrix $Q^{A B}$ which corresponds to the $I$-th piece of the graph $\Gamma_{p}$ shown in Figure 3 is represented as

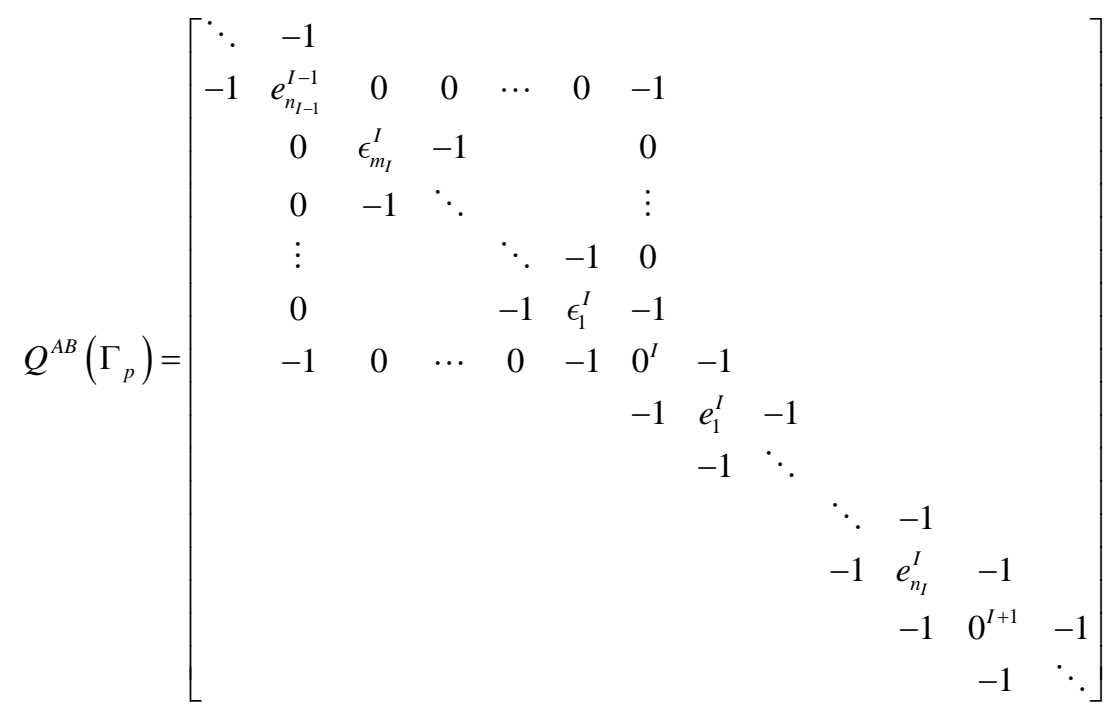

Note that $0^{I}$ denotes an integer number 0 corresponding to the node $N^{I}$. Now we pay attention to the tridiagonal submatrices (blocks) of type

$$
B^{a b}=\left(\begin{array}{cccccc}
0 & -1 & & & & \\
-1 & e_{1} & -1 & & & \\
& -1 & \ddots & & & \\
& & & \ddots & -1 & \\
& & & -1 & e_{n} & -1 \\
& & & & -1 & 0
\end{array}\right)
$$

and notice that using Gauss-Neumann partial diagonalization [7] the matrix is equivalent to the rational block matrix

$$
\left[e_{1}\right] \oplus\left[e_{2}, e_{1}\right] \oplus \cdots \oplus\left[e_{n}, \cdots, e_{1}\right] \oplus B_{\text {red }}
$$

where 


$$
B_{\mathrm{red}}=-\left(\begin{array}{cc}
\frac{q}{p} & \frac{1}{p} \\
\frac{1}{p} & \frac{q^{*}}{p}
\end{array}\right)
$$

and $\frac{p}{q}=-\left[e_{1}, \cdots, e_{n}\right], \frac{p}{q^{*}}=-\left[e_{n}, \cdots, e_{1}\right], p=-\left[e_{1}\right]\left[e_{2}, e_{1}\right]\left[e_{3}, e_{2}, e_{1}\right] \cdots\left[e_{n}, \cdots, e_{1}\right]$. Here we are using the standard definition of continued fraction

$$
\left[e_{1}, \cdots, e_{n}\right]=e_{1}-\frac{1}{e_{2}-\frac{1}{\cdots-\frac{1}{e_{n}}}} .
$$

Applying the general Gauss-Neumann partial diagonalization method for the matrix $Q^{A B}\left(\Gamma_{p}\right)$ we obtain a similar result where $Q_{\text {red }}$ is a rational tridiagonal matrix of rank $R$ (the number of nodes of the graph $\Gamma_{p}$ ) whose elements on the diagonal are a sum of three terms representing each maximal chain connecting to the node.

$$
Q_{\mathrm{red}}^{I J}\left(\Gamma_{p}\right)=- \begin{cases}\frac{b^{0}}{a^{0}}+\frac{q^{1}}{p^{1}}+\frac{b^{1}}{a^{1}}, & \text { if } I=J=1 ; \\ \frac{q^{* I-1}}{p^{I-1}}+\frac{q^{I}}{p^{I}}+\frac{b^{I}}{a^{I}}, & \text { if } I=J=2, \cdots, R-1 ; \\ \frac{q^{R-1}}{p^{R-1}}+\frac{b^{R}}{a^{R}}+\frac{b^{R+1}}{a^{R+1}}, & \text { if } I=J=R \\ 0, & \text { if } J=I-1 \text { or } J=I+1 \\ & \text { other case }\end{cases}
$$

where $\frac{a^{I}}{b^{I}}=-\left[\epsilon_{1}^{I}, \cdots, \epsilon_{m_{I}}^{I}\right]$ are continued fractions for a terminal chain, and $\frac{p^{I}}{q^{I}}=-\left[e_{1}^{I}, \cdots, e_{n_{I}}^{I}\right]$ for a internal chain. We have used the notation $q^{*}$ to indicate that the order of the numbers on the continued fraction is inverse, i.e. $\frac{p^{I}}{q^{* I}}=-\left[e_{n_{I}}^{I}, \cdots, e_{1}^{I}\right]$. So, we can reduce each chain of $\Gamma_{p}$ to a rational number $\frac{b}{a}$, $\frac{q}{p}$ or $\frac{q^{*}}{p}$ which is represented as a continued fraction, and thus reduce the original block tridiagonal matrix $Q\left(\Gamma_{p}\right)$ to a tridiagonal matrix $Q_{\text {red }}\left(\Gamma_{p}\right)$ whose size depends just of the number of nodes of $\Gamma_{p}$. It is important to note that it is possible to obtain the original matrix $Q\left(\Gamma_{p}\right)$ from the reduced matrix $Q_{\text {red }}\left(\Gamma_{p}^{p}\right)$.

\section{Rational Linking Matrix for Graph Manifold $M\left(\Gamma_{p}\right)$}

In this section we will construct a plumbing graph manifold $M\left(\Gamma_{p}\right)$ codified by the same graph $\Gamma_{p}$ as in section 2. Now we see the weight $e_{i}$ as the Euler number of the principal $S^{1}$-(U(1)-)bundle, corresponding to $i$-th vertex $v_{i}$. We define the bundle $M\left(e_{i}\right)$ associated to each vertex $v_{i}$ as $S^{1}$-bundle over $S^{2}$ with the Euler number $e_{i}$, which can be pasted together from two trivial bundles over $D^{2}$ as follows [8] [9]

$$
D^{2} \times S^{1} \cup_{H_{i}} D^{2} \times S^{1}, H_{i}: \partial\left(D^{2} \times S^{1}\right)=S^{1} \times S^{1} \rightarrow S^{1} \times S^{1}=\partial\left(D^{2} \times S^{1}\right)
$$

where

$$
H_{i}\left(\begin{array}{l}
x \\
y
\end{array}\right)=\left(\begin{array}{ll}
-1 & 0 \\
-e_{i} & 1
\end{array}\right)\left(\begin{array}{l}
x \\
y
\end{array}\right)=\left(\begin{array}{l}
-x \\
y-e_{i} x
\end{array}\right) .
$$


Note that the above is a well known description of the lens space $L\left(e_{i}, 1\right)$, so the total space of the bundle is $M\left(e_{i}\right)=L\left(e_{i}, 1\right)$. To perform pasting operation, which is known as plumbing between the $S^{1}$-bundles, we must use the trivial bundles over annuli $M_{A}\left(e_{i}\right)=A \times S^{1} \cup_{H_{i}} A \times S^{1}$ where $A$ is an annulus or twice punctured sphere $S^{2}$. The manifold $M\left(\Gamma_{p}\right)$ is pasted together from the manifolds $M_{A}\left(e_{i}\right)$ as follows [9]: whenever vertices $v_{i}$ and $v_{j}$ are connected by an edge $\sigma_{i j}$ in $\Gamma_{p}$ we paste a boundary component $S^{1} \times S^{1}$ of $M_{A}\left(e_{i}\right)$ to a boundary component $S^{1} \times S^{1}$ of $M_{A}\left(e_{j}\right)$ by the map $J=\left(\begin{array}{ll}0 & 1 \\ 1 & 0\end{array}\right)$ :

$A \times S^{1} \cup_{H_{i}} A \times S^{1} \cup_{J} A \times S^{1} \cup_{H_{j}} A \times S^{1}$, so the base and fiber coordinates are exchanged under the plumbing operation. Thus the edge $\sigma_{i j}$ corresponds to the torus $T_{i j}^{2}=S^{1} \times S^{1}$ along which the pieces $M_{A}\left(e_{i}\right)$ and $M_{A}\left(e_{j}\right)$ pasted together.

For example, the plumbing of the chain shown in Figure 1 gives us the pasting

$$
M\left(N^{I}\right) \cup_{J}\left(A \times S^{1} \cup_{H_{i}} A \times S^{1}\right) \cup_{J} \cdots \cup_{J}\left(A \times S^{1} \cup_{H_{k}} A \times S^{1}\right) \cup_{J} M\left(N^{J}\right),
$$

where $M\left(N^{I}\right)$ is a $S^{1}$-bundle associating with the node $N^{I}$. This chain corresponds to a Seifert fibered thick torus (homeomorphic to $\left.T^{2} \times[0,1]\right)$ in graph manifold $M\left(\Gamma_{p}\right)$. Also, the terminal maximal chain shown in Figure 2 corresponds to a Seifert fibered solid torus (homeomorphic to $\left.D^{2} \times S^{1}\right)$ in $M\left(\Gamma_{p}\right)$. The using of graphs with nodes of valence $n=3$ (as in Section 2) corresponds to plumbing of Brieskorn homology spheres (Bh-spheres) [4].

Now recall that each edge $\sigma$ of $\Gamma_{p}$ relates to the embedded torus $T_{\sigma}^{2} \subset M\left(\Gamma_{p}\right)$ and the collection of all these tori cuts the graph manifold $M\left(\Gamma_{p}^{p}\right)$ into disjoint union of circle bundles over $n$ times punctured sphere $S^{2} \backslash \bigcup_{I=1}^{n} p_{l}, 1 \leq n \leq 3$. In general, the bundles are over compact surfaces of genus $g$ with some boundary components, see [8] [9]. Such a collection of tori $\mathcal{T}_{W}$ is called a graph structure on $M\left(\Gamma_{p}\right)$ by Waldhausen [10]. We want to define the Jaco-Shalen-Johannson (JSJ) graph structure $\mathcal{T}_{\text {JSJ }} \subset \mathcal{T}_{W}$ of the Waldhausen graph structure and to specify the corresponding JSJ-decomposition of graph manifold $M\left(\Gamma_{p}\right)$ on the set of Seifert fibered pieces $M_{\mathrm{JSJ}}\left(N^{I}\right)$. Let us denote $\mathcal{C}(p)$ the set of maximal chains in the graph $\Gamma_{p}$. This set can be written as a disjoint union $\mathcal{C}(p)=\mathcal{C}_{i}(p) \sqcup \mathcal{C}_{t}(p)$, where $\mathcal{C}_{i}(p)$ denote the set of interior chains and $\mathcal{C}_{t}(p)$ is the set of terminal chains. The edges of $\Gamma_{p}$ contained in a chain $C \in \mathcal{C}(p)$ correspond to a set of parallel tori in $M\left(\Gamma_{p}\right)$. Choose one torus $T_{C}^{2}$ among them and define

$$
\mathcal{T}_{\text {JSJ }}=\bigsqcup_{C \in \mathcal{C}_{i}(p)} T_{C}^{2}
$$

This set of tori performs the well known JSJ-decomposition of the graph manifold $M\left(\Gamma_{p}\right)$ [11].

By construction, each piece $M_{\mathrm{JSJ}}\left(N^{I}\right)$ (denoted as $M_{\mathrm{JSJ}}^{I}$ for brevity) of JSJ-decomposition that corresponds to the node $N^{I}$ contains a unique piece $M_{W}\left(N^{I}\right)$ (which we shall denote as $M_{W}^{I}$ ) of Waldhausen decomposition associated with the same node $N^{I}$. One can extend in a unique way up to isotopy the natural Seifert structure without exceptional fibers on $M_{W}^{I}$ to a Seifert fibration on $M_{\mathrm{JSJ}}^{I}$ with exceptional fibers. Thus in these terms the JSJ-decomposition of the manifold $M\left(\Gamma_{p}\right)$ is defined completely by $M\left(\Gamma_{p}\right)=\bigcup_{I=1}^{R} \bar{M}_{J S J}^{I}$, where $R$ is the number of nodes in $\Gamma_{p}$ and the bar over $M$ means the closure of the piece $M_{\mathrm{JSJ}}^{I}$.

Note that there exists an uncertainty in the choice of the torus $T_{C}^{2}$ for each internal chain which appear in the JSJ-structure (2). We can remove this uncertainty in following way. Let us perform the maximal extension of the natural Seifert fibration from each $M_{W}^{I}$ and denote the obtained Seifert fibered piece of $M\left(\Gamma_{p}\right)$ by $\hat{M}^{I}$. It is clear that $\hat{M}^{I} \cap \hat{M}^{J} \neq \varnothing$ if and only if there exists a chain $C_{I J}$ joining the nodes $N^{I}$ and ${ }^{p} N^{J}$. If we start with plumbing of $R$ Bh-spheres $\left\{\Sigma\left(a_{1}^{I}, a_{2}^{I}, a_{3}^{I}\right) \mid I=1, \cdots, R\right\}$, the resulting graph three-manifold will be integer homology sphere [4] [9] (ZZ-homology sphere), which in general case does not have the global Seifert fibration. But we can construct the JSJ-covering $\mathcal{M}:=\left\{\hat{M}^{I} \mid I=1, \cdots, R\right\}$, such as each $\hat{M}^{I}$ is a Seifert fibered space and it is maximal in the sense described above.

Suppose that we perform the plumbing operation according to the plumbing diagram $\Delta_{p}$, shown on the Figure 4. Thus our plumbing diagrams will always have the pairwise coprime weights around each node and correspond to $\mathbb{Z}$-homology spheres [5].

We construct the plumbing graph $\Gamma_{p}$ for a $\mathbb{Z}$-homology sphere, following [5] [8] [9] (as a result we shall obtain a graph of type shown in Figure 3). First of all we calculate the characteristics of maximal chains. For terminal chains the integer Euler numbers $\epsilon_{i}^{I}$ are defined by the continued fraction: 


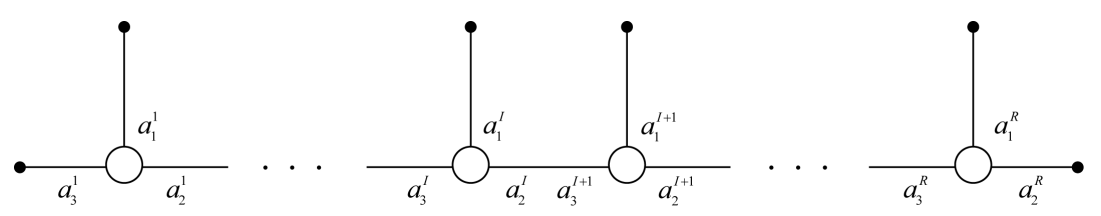

Figure 4. A plumbing (splicing) diagram $\Delta_{p}$.

$$
-\frac{a^{I}}{b^{I}}=\left[\epsilon_{1}^{I}, \cdots, \epsilon_{m_{I}}^{I}\right]
$$

where $\left(a^{I}, b^{I}\right), \quad I=0, \cdots, R+1$ are the Seifert invariants, numerated in the following way

$$
a^{0}=a_{3}^{1}, b^{0}=b_{3}^{1} ; a^{J}=a_{1}^{J}, b^{J}=b_{1}^{J}, J=1, \cdots, R ; a^{R+1}=a_{2}^{R}, b^{R+1}=b_{2}^{R} .
$$

For internal chains the integer Euler numbers $e_{i}^{I}$ are defined by

$$
-\frac{p^{I}}{q^{I}}=\left[e_{1}^{I}, \cdots, e_{n_{I}}^{I}\right]
$$

where the Seifert (orbital) invariants $\left(p^{I}, q^{I}\right), I=1, \cdots, R-1$ characterize the thick tori $T T\left(p^{I}, q^{I}\right) \cong T^{2} \times[0,1]$, which are created by the plumbing operations performed between the nodes $N^{I}$ and $N^{I+1}$, see [6] [9]. These invariants identify also the extra lens spaces $L\left(p^{I}, q^{I}\right)$ which arise in four-dimensional plumbed V-cobordism (corresponding to the graph $\Gamma_{p}$ ) with

$$
p^{I}=a_{2}^{I} a_{3}^{I+1}-a_{1}^{I} a_{3}^{I} a_{1}^{I+1} a_{2}^{I+1}, \quad q^{I}=b_{2}^{I} a_{3}^{I+1}+a_{1}^{I+1} a_{2}^{I+1}\left(b_{1}^{I} a_{3}^{I}+a_{1}^{I} b_{3}^{I}\right)
$$

for the ordering fixed by the plumbing diagram in Figure 4. From this representation of the plumbing graph it is clear that for $I=2, \cdots, R-1$ the set $\hat{M}^{I}$ of JSJ-covering $\mathcal{M}$ has the form

$$
\hat{M}^{I}=\bar{M}_{W}^{I} \cup T T\left(p^{I-1}, q^{* I-1}\right) \cup T T\left(p^{I}, q^{I}\right) \cup S T\left(a^{I}, b^{I}\right),
$$

where $S T\left(a^{I}, b^{I}\right)$ is a Seifert fibered solid torus with Seifert invariants $\left(a^{I}, b^{I}\right)$ and

$$
-\frac{p^{I}}{q^{* I}}=\left[e_{n_{I}}^{I}, \cdots, e_{1}^{I}\right]
$$

For the cases $I=1$ and $I=R$ the formulas are different from (6):

$$
\begin{gathered}
\hat{M}^{1}=\bar{M}_{W}^{I} \cup T T\left(p^{1}, q^{1}\right) \cup S T\left(a^{0}, b^{0}\right) \cup S T\left(a^{1}, b^{1}\right), \\
\hat{M}^{R}=\bar{M}_{W}^{R} \cup T T\left(p^{R-1}, q^{* R-1}\right) \cup S T\left(a^{R}, b^{R}\right) \cup S T\left(a^{R+1}, b^{R+1}\right) .
\end{gathered}
$$

Moreover $\hat{M}^{I} \cap \hat{M}^{I+1}=T T\left(p^{I}, q^{I}\right) \cong^{*} T T\left(p^{I}, q^{* I}\right) I=2, \cdots, R-1$. Here the symbol $\cong^{*}$ indicates that $T T\left(p^{I}, q^{I}\right)$ and $T T\left(p^{I}, q^{* I}\right)$ are homeomorphic, but their Seifert structures are characterized by different integer Euler numbers defined by (5) and (7) respectively. Thereby the thick torus between the nodes $N^{I}$ and $N^{I+1}$ has two Seifert fibrations: the first is the extension of the natural Seifert fibration defined on the piece $M_{W}^{I}$ and the second one is obtained as extension from the piece $M_{W}^{I+1}$. These Seifert fibrations are connected by the matrix [6] [9]:

$$
S^{I}=\left(\begin{array}{cc}
-q^{I} & p^{* I} \\
p^{I} & q^{* I}
\end{array}\right)=\left(\begin{array}{ll}
-1 & 0 \\
-e_{1} & 1
\end{array}\right)\left(\begin{array}{cc}
0 & 1 \\
1 & 0
\end{array}\right)\left(\begin{array}{cc}
-1 & 0 \\
-e_{2} & 1
\end{array}\right) \ldots\left(\begin{array}{cc}
0 & 1 \\
1 & 0
\end{array}\right)\left(\begin{array}{ll}
-1 & 0 \\
-e_{n} & 1
\end{array}\right)
$$

in the following sense. Recall that edges of $\Gamma_{p}$ contained in a chain $C_{I, I+1}$ (between the nodes $N^{I}$ and $N^{I+1}$ ) correspond to the set parallel tori in $M\left(\Gamma_{p}\right)$. On any of these tori there exist two bases formed by the section lines and the fibers pertain to the Seifert fibrations extended from $M_{W}^{I}$ and $M_{W}^{I+1}$, which we denote as the pair of columns

$$
\left(\begin{array}{l}
s_{2}^{I} \\
f_{I}
\end{array}\right) \text { and }\left(\begin{array}{l}
s_{3}^{I+1} \\
f_{I+1}
\end{array}\right)
$$


Subindices 2 and 3 manifest that $\Sigma\left(a_{1}^{I}, a_{2}^{I}, a_{3}^{I}\right)$ and $\Sigma\left(a_{1}^{I+1}, a_{2}^{I+1}, a_{3}^{I+1}\right)$ are plumbed together along the singular fibers with Seifert invariants $a_{2}^{I}$ and $a_{3}^{I+1}$ (see Figure 4). Then the transformation between these section-fiber bases is described by

$$
\left(\begin{array}{l}
s_{2}^{I} \\
f_{I}
\end{array}\right)=\left(\begin{array}{cc}
-q^{I} & p^{* I} \\
p^{I} & q^{* I}
\end{array}\right)\left(\begin{array}{c}
s_{3}^{I+1} \\
f_{I+1}
\end{array}\right),
$$

where $p^{* I}$ is defined from $\operatorname{det} S^{I}=-\left(q^{I} q^{* I}+p^{I} p^{* I}\right)=-1$.

Now we introduce the one-form bases $\left(\sigma_{I}^{2}, \kappa^{I}\right)$ and $\left(\sigma_{I+1}^{3}, \kappa^{I+1}\right)$, duals to the bases (10) in the following sense:

$$
\int_{s_{2}} \sigma_{I}^{2}=\int_{s_{3}+1} \sigma_{I+1}^{3}=\int_{f_{I}} \kappa^{I}=1 ; \int_{f_{I}} \sigma_{I}^{2}=\int_{f_{I+1}} \sigma_{I+1}^{3}=\int_{s_{2}^{I}} \kappa^{I}=\int_{s_{3}+1} \kappa^{I+1}=0,
$$

where the integrals are calculated over any such section line or fiber as, for example, in [12]. Thus we obtain the corresponding transformations between the the dual one-forms:

$$
\sigma_{I}^{2}=-q^{* I} \sigma_{I+1}^{3}+p^{I} \kappa^{I+1} ; \quad \kappa^{I}=p^{* I} \sigma_{I+1}^{3}+q^{I} \kappa^{I+1} .
$$

We suppose that the forms $\sigma$ and $\kappa$ are dual with respect to the bilinear pairing defined as

$$
\left\langle\sigma_{I}^{2}, \kappa^{J}\right\rangle:=\int_{\hat{M}^{I} \cap \hat{M}^{J}} \sigma_{I}^{2} \wedge \mathrm{d} \kappa^{J}=\delta_{I}^{J} ;\left\langle\sigma_{I+1}^{3}, \kappa^{J}\right\rangle:=\int_{\hat{M}^{I+1} \cap \hat{M}^{J}} \sigma_{I+1}^{3} \wedge \mathrm{d} \kappa^{J}=\delta_{I+1}^{J},
$$

Also we shall used the integrals

$$
\Lambda^{I, I+1}=\int_{T T\left(p^{I}, q^{I}\right)} \kappa^{I} \wedge \mathrm{d} \kappa^{I+1} ; \quad \Lambda^{I, I}=\int_{T T\left(p^{I}, q^{I}\right)} \kappa^{I} \wedge \mathrm{d} \kappa^{I} ; \quad \Lambda^{I+1, I+1}=\int_{T T\left(p^{I}, q^{* I}\right)} \kappa^{I+1} \wedge \mathrm{d} \kappa^{I+1},
$$

which define the linking (intersection) numbers of the fiber structures $\kappa^{I}$ and $\kappa^{I+1}$ defined on thick torus $T T\left(p^{I}, q^{I}\right) \cong^{*} T T\left(p^{I}, q^{* I}\right)$. We can obtain the rational linking matrix for $T T\left(p^{I}, q^{I}\right) \cong^{*} T T\left(p^{I}, q^{* I}\right)$ by means of multiplication of the Equations (11) by $\mathrm{d} \kappa^{I}$ and $\mathrm{d} \kappa^{I+1}$ and integration over $T T\left(p^{I}, q^{I}\right) \cong^{*} T T\left(p^{I}, q^{* I}\right)$. Applying the duality conditions (12) we obtain:

$$
\Lambda^{I, I+1}=\Lambda^{I+1, I}=\frac{1}{p^{I}} \Lambda^{I, I}=\frac{q^{I}}{p^{I}} ; \quad \Lambda^{I+1, I+1}=\frac{q^{* I}}{p^{I}} .
$$

The rational numbers $\Lambda^{I, I}$ and $\Lambda^{I+1, I+1}$ are also known as Chern classes of the line V-bundles associated with the Seifert fibrations with the $U(1)$-invariant connection forms $\kappa^{I}$ and $\kappa^{I+1}$ on the lens spaces $L\left(p^{I}, q^{I}\right)$ and $L\left(p^{I}, q^{* I}\right)$ respectively [12].

Now we are ready to calculate the rational linking matrix for the graph manifold $-M\left(\Gamma_{p}\right)$ (see Figure 3):

$$
K^{I J}=-\int_{M\left(\Gamma_{p}\right)} \kappa^{I} \wedge \mathrm{d} \kappa^{J} .
$$

We integrate here over the three dimensional graph manifold $-M\left(\Gamma_{p}\right)$ to obtain a positive definite linking matrix. This manifold has the opposite orientation with respect to the graph manifold $M\left(\Gamma_{p}\right)$ obtained directly by plumbing of Bh-spheres, which are defined as links of singularities. This construction of the graph manifold $M\left(\Gamma_{p}\right)$ gives the possibility to represent it also as a link of singularity that guarantees its rational linking matrix to be negative definite (for details see [5]).

From the tree structure of the graph $\Gamma_{p}$, and from the first equation in (13) we immediately obtain, that for $I \neq J$ the nonzero elements are only

$$
K^{I, I+1}=K^{I+1, I}=-\int_{\hat{M}^{I} \cap \hat{M}^{I+1}} \kappa^{I} \wedge \mathrm{d} \kappa^{I+1}=-\int_{T T\left(p^{I}, q^{I}\right)} \kappa^{I} \wedge \mathrm{d} \kappa^{I+1}=-\frac{1}{p^{I}},
$$

for $1 \leq I \leq R-1$.

If $I=J=2, \cdots, R-1$, we have

$$
K^{I I}=-\int_{\hat{M}^{I}} \kappa^{I} \wedge \mathrm{d} \kappa^{I}=-\int_{T T\left(p^{I-1}, q^{* I-1}\right)} \kappa^{I} \wedge \mathrm{d} \kappa^{I}-\int_{T T\left(p^{I}, q^{I}\right)} \kappa^{I} \wedge \mathrm{d} \kappa^{I}-\int_{S T\left(a^{I}, b^{I}\right)} \kappa^{I} \wedge \mathrm{d} \kappa^{I} .
$$

Here we use the decomposition (6) of the piece $\hat{M}^{I}$, and that the integral over trivial Seifert fibration $\bar{M}_{W}^{I}$ is zero. Then according to the two last equations in (13) we obtain the matrix element 


$$
K^{I I}=-\left(\frac{q^{* I-1}}{p^{I-1}}+\frac{q^{I}}{p^{I}}+\frac{b^{I}}{a^{I}}\right),
$$

also known as the Chern class of line $V$-bundle associated with the Seifert fibration of $\hat{M}^{I}$.

For $I=1$ and $I=R$ the matrix elements are

$$
\begin{gathered}
K^{11}=-\int_{\hat{M}^{1}} \kappa^{I} \wedge \mathrm{d} \kappa^{I}=-\int_{S T\left(a^{0}, b^{0}\right)} \kappa^{1} \wedge \mathrm{d} \kappa^{1}-\int_{T T\left(p^{1}, q^{1}\right)} \kappa^{1} \wedge \mathrm{d} \kappa^{1}-\int_{S T\left(a^{1}, b^{1}\right)} \kappa^{I} \wedge \mathrm{d} \kappa^{I} ; \\
K^{R R}=-\int_{\hat{M}^{R}} \kappa^{R} \wedge \mathrm{d} \kappa^{R}=-\int_{T T\left(p^{R-1}, q^{* R-1}\right)} \kappa^{R} \wedge \mathrm{d} \kappa^{R}-\int_{S T\left(a^{R}, b^{R}\right)} \kappa^{R} \wedge \mathrm{d} \kappa^{R}-\int_{S T\left(a^{R+1}, b^{R+1}\right)} \kappa^{R} \wedge \mathrm{d} \kappa^{R} ; \\
K^{11}=-\left(\frac{b^{0}}{a^{0}}+\frac{q^{1}}{p^{1}}+\frac{b^{1}}{a^{1}}\right) ; \quad K^{R R}=-\left(\frac{q^{* R-1}}{p^{R-1}}+\frac{b^{R}}{a^{R}}+\frac{b^{R+1}}{a^{R+1}}\right) .
\end{gathered}
$$

Here we have used the decompositions (8) and (9) as well as the notations (4).

\section{Conclusions}

Comparing the reduced matrix $Q_{\text {red }}\left(\Gamma_{p}\right)$ (1) with the results (14) and (17) for the rational linking matrix $K$ of the graph manifold $-M\left(\Gamma_{p}\right)$ we observe that decomposing the rational invariants into continued fractions according to (3), (5) and (7), we can create the graph $\Gamma_{p}$ (related to diagram $\Delta_{p}$ ) and obtain the rational linking matrix $K$ of $-M\left(\Gamma_{p}\right)$ just by Gauss-Neumann diagonalization on the Laplacian matrix of $\Gamma_{p}$. This is the main result of this article.

We want to conclude with an example of an application of our results for the topological field theory. In [3] [13] we built a set of graph manifolds whose Seifert invariants are constructed on the base of the first 9 prime numbers $p_{1}=2, p_{2}=3, \cdots, p_{9}=23$. The rational linking matrix of these graph manifolds are positive definite and have diagonal elements (and eigenvalues) simulating the low-energy coupling constants hierarchy of the fundamental interactions of real universe. An example of such matrix is [13]

$$
K^{I J}=\left(\begin{array}{ccccc}
9.69 \times 10^{-1} & -3.13 \times 10^{-2} & 0 & 0 & 0 \\
-3.13 \times 10^{-2} & 7.21 \times 10^{-3} & -1.44 \times 10^{-8} & 0 & 0 \\
0 & -1.44 \times 10^{-8} & 1.76 \times 10^{-12} & -1.93 \times 10^{-29} & 0 \\
0 & 0 & -1.93 \times 10^{-29} & 3.68 \times 10^{-44} & -3.12 \times 10^{-89} \\
0 & 0 & 0 & -3.12 \times 10^{-89} & 2.66 \times 10^{-134}
\end{array}\right) \text {, }
$$

whose elements are all rational and the diagonal ones are described in (14) and (17) by a sum of three continued fractions. The matrix $K_{I J}$ inverse to $K^{I J}$ is integer one [6], the inversion of the rational linking matrices can be done with the help of any program such as MathematicaTM to verify this property, any error on calculation of $K^{I J}$ leads to non-integer elements in resulting matrices $K_{I J}$. It is also worth mentioning that in the present example the Laplacian block matrix $Q^{A B}\left(\Gamma_{p}\right)$, corresponding to the matrix $K^{I J}=Q_{\text {red }}^{I J}\left(\Gamma_{p}\right)$, has $\operatorname{rank}\left(Q^{A B}\left(\Gamma_{p}\right)\right) \approx 4.019 \times 10^{20} \quad[13]$, while $\operatorname{rank}\left(K^{I J}\right)=5$.

In the 7-dimensional Kaluza-Klein approach to the topological field theory (BF-model), the rational linking matrices of the 3-dimensional graph manifold may be really interpreted as coupling constants matrices [1]. So, the Gauss-Neumann method gives us a simple algorithm to calculate the linking matrices of graph manifolds and thus the coupling constants matrices (despite the probably huge rank of the original block matrix $Q^{A B}\left(\Gamma_{p}\right)$ ).

\section{References}

[1] Verlinde, E. (1995) Global Aspects of Electric-Magnetic Duality. Nuclear Physics B, 455, 211-225. http://dx.doi.org/10.1016/0550-3213(95)00431-Q

[2] Efremov, V.N., Mitskievich, N.V., Hernandez Magdaleno, A.M. and Serrano Bautista, R. (2005) Topological Gravity on Plumbed V-Cobordisms. Classical and Quantum Gravity, 22, 3725-3744. http://dx.doi.org/10.1088/0264-9381/22/17/022

[3] Efremov, V.N., Hernandez Magdaleno, A.M. and Moreno, C. (2010) Topological Origin of the Coupling Constants Hierarchy in Kaluza-Klein Approach. International Journal of Modern Physics A, 25, 2699-2733. 
http://dx.doi.org/10.1142/S0217751X10048482

[4] Saveliev, N. (2002) Invariants for Homology3-Spheres. Springer, Berlin, 223. http://dx.doi.org/10.1007/978-3-662-04705-7

[5] Eisenbud, D. and Neumann, W. (1985) Three-Dimensional Link Theory and Invariants of Plane Curve Singularities. Princeton University Press, Princeton, 172.

[6] Saveliev, N. (2002) Fukumoto-Furuta Invariants of Plumbed Homology 3-Spheres. Pacific Journal of Mathematics, 205, 465-490. http://dx.doi.org/10.2140/pjm.2002.205.465

[7] Neumann, W. (1997) Commensurability and Virtual Fibration for Graph Manifolds. Topology, 36, 355-378. http://dx.doi.org/10.1016/0040-9383(96)00014-6

[8] Hirzebruh, F. (1971) Differentiable Manifolds and Quadratic Forms. Marcel Dekker, New York, 56-58.

[9] Neumann, W. (1981) A Calculus for Plumbing Applied to the Topology of Complex Surface Singularities and Degenerating Complex Curves. Transactions of the American Mathematical Society, 268, 299. http://dx.doi.org/10.1090/S0002-9947-1981-0632532-8

[10] Waldhausen, F. (1967) Eine Klasse Von 3-Dimensionalen Mannigfaltigkeiten. I. Inventiones Mathematicae, 3, 308333. http://dx.doi.org/10.1007/BF01402956

[11] Popescu-Pampu, P. (2007) The Geometry of Continued Fractions and the Topology of Surface Singularities. In: Brasselet, J.-P. and Suwa, T., Eds., Singularities in Geometry and Topology 2004, Advanced Studies in Pure Mathematics, Vol. 46, 119-195.

[12] Beasley, C. and Witten, E. (2005) Non-Abelian Localization for Chern-Simons Theory. Journal of Differential Geometry, 70, 183-323.

[13] Efremov, V., Hernandez, A. and Becerra, F. (2014) The Universe as a Set of Topological Fluids with Hierarchy and Fine Tuning of Coupling Constants in Terms of Graph Manifolds. arXiv:1309.0690v2 
Scientific Research Publishing (SCIRP) is one of the largest Open Access journal publishers. It is currently publishing more than 200 open access, online, peer-reviewed journals covering a wide range of academic disciplines. SCIRP serves the worldwide academic communities and contributes to the progress and application of science with its publication.

Other selected journals from SCIRP are listed as below. Submit your manuscript to us via either submit@scirp.org or Online Submission Portal.
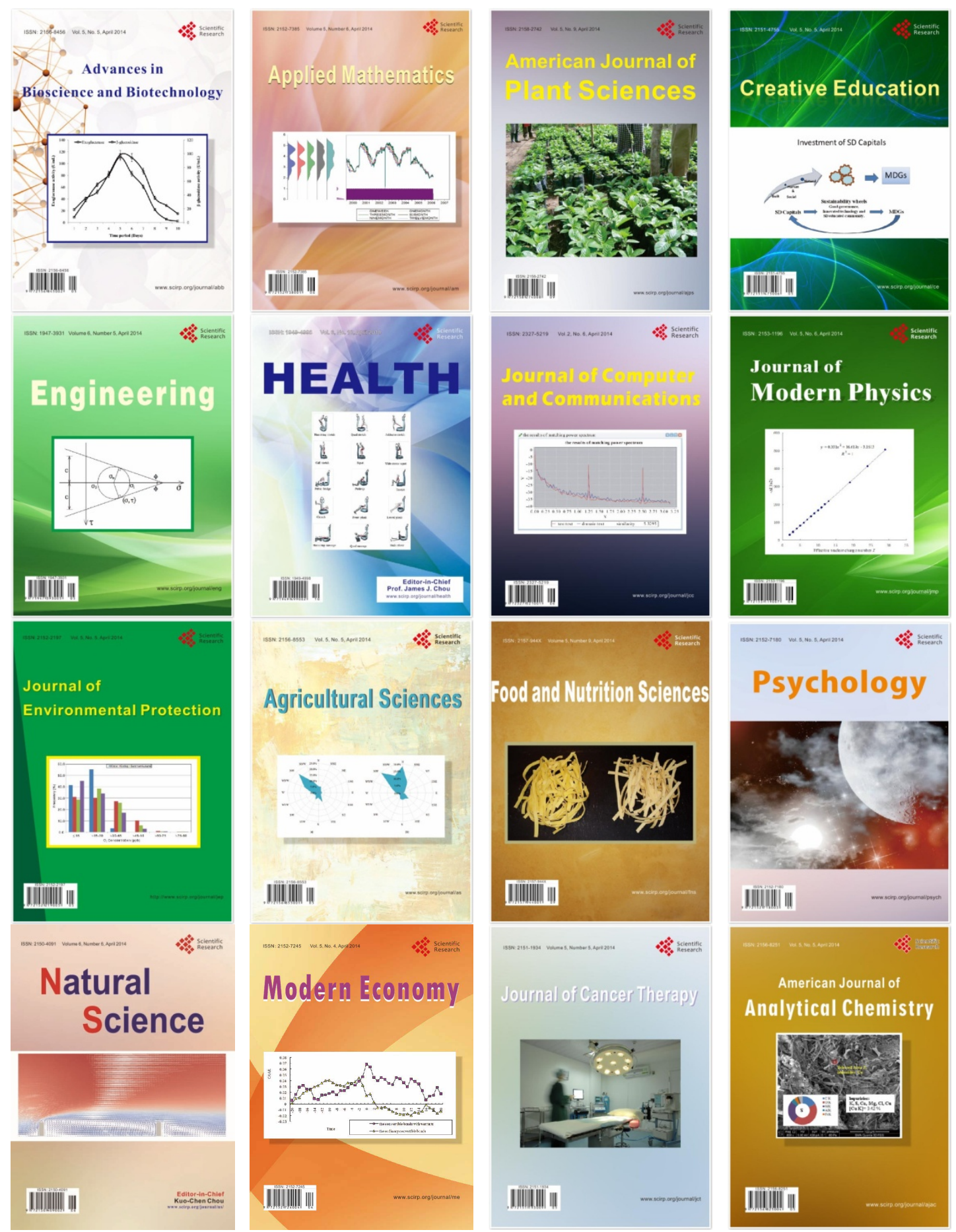